\title{
SPLITTING OF GEODESICS IN HOMOLOGY CLASSES
}

\author{
STEVEN ZELDITCH
}

(Communicated by David G. Ebin)

\begin{abstract}
We prove a Chebotarev density theorem for closed geodesics in a fixed homology class on a compact hyperbolic surface. The theorem (and its proof) combines some work of Adachi-Sunada and Phillips-Sarnak.
\end{abstract}

The analogy between the splitting of closed geodesics in a finite Riemannian covering $\pi: M \rightarrow M_{0}$ and the splitting of prime ideals in field extensions has been firmly established by a number of authors (e.g. [Sa], [Su]). In particular one has a Tchebotarev density theorem for prime closed geodesics (or, primes) in Galois covers of finite volume hyperbolic manifolds. This asserts, roughly speaking, that the probability of a prime $\rho$ of $M_{0}$ splitting into $g$ distinct primes $\mathscr{P}_{1}, \ldots, \mathscr{P}_{g}$ (i.e. $\pi^{-1}(\mu)=\bigsqcup_{i=1}^{g} \mathscr{P}_{i}$ ), with given Frobenius class $\left\{\left(\mathscr{P}_{i} \mid \pi\right)\right\}$ is equal to $\left|\left\{\left(\mathscr{P}_{i} \mid \pi\right)\right\}\right| /|G|$. Here $\left(\mathscr{P}_{i} \mid \pi\right)$ generates the stabilizer of $\mathscr{P}_{i}$ in $G,\{\cdot\}$ is its conjugacy class, and $|\cdot|$ denotes cardinality.

The aim of the present paper is to add a footnote to the Tchebotavev density theorem, by combining it with recent work of Phillips-Sarnak and AdachiSunada on closed geodesics in a fixed homology class. The question we pose is: Suppose $M_{0}$ is a compact hyperbolic surface, and suppose we fix a homology class $A \in H_{1}\left(M_{0}, \mathbb{Z}\right)$. What is the probability that a prime in $A$ splits into $\mathscr{P}_{1}, \ldots, \mathscr{P}_{g}$ in $M$, with Frobenius class $\left\{\left(\mathscr{P}_{i} \mid \pi\right)\right\}$, in a galois (i.e., normal) cover $\pi: \stackrel{g}{M} \rightarrow M_{0}$.

As the reader will note at once, the answer depends on the galois (i.e., deck transformation) group $G$. If $G$ is Abelian, then the splitting type is an invariant of $A$. If on the other hand $G$ is perfect, then one may expect (correctly) that the splitting type of primes in $A$ is completely random. In fact, the result is (see $\S 1.6)$ :

Theorem. With the above hypothesis, the probability that the Frobenius class $\mathrm{Fr}=\operatorname{def}\left\{\left(\mathscr{P}_{i} \mid \pi\right)\right\}$ is a given conjugacy class $C$ equals: $|G /[G, G]|$ $\times|C| /|G| \delta(A, C, G /[G, G])$, where $\delta(A, C, G /[G, G])$ equals 1 if $A$ and $C$ project to the same element in $G /[G, G]$, and is 0 otherwise.

Received by the editors December 2, 1987 and, in revised form, June 20, 1988.

1980 Mathematics Subject Classification (1985 Revision). Primary 58E10, 58F17. 
The proof is simple, given the ideas of the aforementioned papers. It will be given in $\S 1$. The statement of the theorem makes sense and is no doubt true in the variably curved case, but the proof given here will not generalize (based as it is on the trace formula, as in [PS]).

We will take for granted the results of [PS], and $\left[\mathrm{Su}_{1}, \mathrm{Su}_{2}\right]$, our basic references for this paper.

\section{Proof of the theorem}

To fix notation, we set:

$$
\begin{aligned}
M_{0}= & \Gamma_{0} \mid \mathfrak{h}\left(\mathfrak{h}=\text { upper } \frac{1}{2} \text { plane } ; \Gamma_{0} \subset \operatorname{PSL}_{2}(\mathbb{R})\right. \text { is a discrete co-compact } \\
& \text { subgroup }) .
\end{aligned}
$$

$$
M=\Gamma \mid \mathfrak{h} ; \Gamma \subset \Gamma_{0} \text { is assumed a normal subgroup . }
$$

$$
G=\Gamma \backslash \Gamma_{0} ; G=\text { deck transformation group of } \pi: M \rightarrow M_{0} .
$$

$$
\begin{aligned}
& \Theta=\operatorname{Hom}(\Gamma, U(1)) ; \Theta \text { is the character or Jacobian variety of } \\
& \Gamma(\text { a } 2 g \text {-dimensional torus }) . \\
& \widehat{G}=(\text { equivalent class of }) \text { irreducible unitary representations } \sigma \text { of } G, \\
& \text { with characters } \chi_{\sigma} \text {. Each } \sigma \in \widehat{G} \text { is in } \operatorname{Hom}(\Gamma, U(n)) / U(n) \text { for some } \\
& \text { (1.5) } \quad n=\operatorname{deg} \sigma, \text { and is trivial on } \Gamma_{0} .
\end{aligned}
$$

We will identify $\Theta=\mathbf{R}^{2 g} / \mathbb{Z}^{2 g}$ in the standard way (see [PS]), and will write $\chi_{\theta}$ as the character associated to $\theta \in \mathbb{R}^{2 g} / \mathbb{Z}^{2 g}$.

Given $\sigma \in \widehat{G}$ and $\theta \in \Theta$, we may form the representation $\sigma \otimes \chi_{\theta}$ of $\Gamma$. The space of this representation is:

$$
V(\sigma, \theta)=\left\{F=\left(\begin{array}{c}
f_{1} \\
\vdots \\
f_{n}
\end{array}\right), f_{i} \in C^{\infty}(\mathfrak{h}), F(\gamma z)=\sigma(\gamma) \chi_{\theta}(\gamma) F(z)\right\} .
$$

Now let $R_{\varphi}$ be any convolution operator, with $\varphi \in C_{0}^{\infty}\left(\operatorname{PSL}_{2}(\mathbb{R})|| K\right)$ (biinvariant under $K)$. Then $R_{\varphi}$ operates on all $V(\sigma, \theta)$, hence gives rise to a trace function:

$$
T(\sigma, \theta)=\left.\operatorname{Tr} R_{\varphi}\right|_{V(\sigma, \theta)} .
$$

The trace formula yields ([Sa])

$$
\text { (TF) } T(\sigma, \theta)=\frac{\operatorname{deg}(\sigma)|\Gamma| h \mid}{2 \pi} \varphi(O)+\sum_{\hat{\gamma} \in \widehat{\Gamma}} \chi_{\theta}(\gamma) \chi_{\sigma}(\gamma) a_{\gamma} \hat{h}\left(L_{\gamma}\right),
$$


where:

(i) $|\Gamma| \mathfrak{h} \mid=$ area of $\Gamma \mid \mathfrak{h}$;

(ii) $R_{\varphi}$ is identified with $h(R), \Delta=-\left(\frac{1}{4}+R^{2}\right)$;

(iii) $a_{\gamma}=\frac{L_{\gamma_{0}}}{\operatorname{sh} L_{\gamma} / 2}($ a conjugacy class invariant $)$;

(iv) $\widehat{\Gamma}=$ conjugacy classes of $\Gamma$;

(v) $\hat{h}=$ Fourier transform of $h$.

We now take the inner product over $\widehat{G} \times \Theta$ of $T(\sigma, \theta)$ with a fixed element of the dual. Namely, let $C$ be a fixed conjugacy class of $G$, and let $\delta_{C}$ be the evaluation function on $\widehat{G}$ :

$$
\delta_{C}(\sigma)=\chi_{\sigma}(C)
$$

Then form, for a homology class $A$ and conjugacy class $C$ :

$$
I(C, A)=\frac{|C|}{|G|} \sum_{\sigma \in \hat{G}} \bar{\chi}_{\sigma}(C) \int_{\boldsymbol{\Theta}} T(\sigma, \theta) e(-\langle A, \theta\rangle) d \theta .
$$

Here we indulge in a little abuse of notation in writing $\chi_{\theta}(A)=e(\langle A, \theta\rangle)$ (cf. [PS]).

From (1.8) we get (in an obvious notation):

$$
\begin{aligned}
& I(C, A)=\delta_{\{e\}}(C) \delta_{0}(A)|\Gamma| \mathfrak{h} \mid \varphi(e) \\
& +\frac{|C|}{|G|} \sum_{\hat{\gamma} \in \hat{\Gamma}} a_{\gamma} \hat{h}\left(L_{\gamma}\right)\left\{\sum_{\sigma \in \hat{G}} \bar{\chi}_{\sigma}(C) \chi_{\sigma}(\gamma) \int_{\Theta} \chi_{\theta}(\gamma) \overline{e(\langle A, \theta\rangle)} d \theta\right\} .
\end{aligned}
$$

By an obvious orthogonality, the $\theta$-integral vanishes unless the homology class $H(\gamma)$ of $\gamma$ equals $A$; and also unless the conjugacy class of $\gamma_{G}$, the image of $\gamma$ under $\Gamma_{0} \rightarrow G$, equals $C$. Equivalently, the Frobenius class $\operatorname{Fr}_{\gamma}$ of $\gamma$ must equal $C$. Since the characteristic function of $C$ is $1_{C}(g)=$ $\left(|C| /|G| \sum_{\sigma \in \hat{G}} \bar{\chi}_{\sigma}(C) \chi_{\sigma}(g)\right.$, we get:

$$
I(C, A)=\delta_{\{e\}}(C) \delta_{0}(A)|\Gamma| \mathfrak{h} \mid \phi(e)+\sum_{\substack{\hat{\gamma} \in \hat{\Gamma} \\ H(\hat{\gamma})=A \\ \mathrm{Fr}_{i}=C}} a_{\gamma} \hat{h}\left(L_{\gamma}\right) .
$$

Next, following [PS] word for word, we let $\hat{h}_{T}$ be the characteristic function of the length interval $[0, T]$, and we smooth it out by appropriate convolution to get $\hat{h}_{T, \epsilon}$. We let $I_{T, \varepsilon}(C, A)$ be the right side of $(1.10)$ with this choice of $h$. By the trace formula (1.10) we get the spectral expansion:

$$
I_{T, \epsilon}(C, A)=\frac{|C|}{|G|} \sum_{r_{1}} \sum_{\sigma \in \hat{G}} \bar{\chi}_{\sigma}(C) \int_{\Theta} h_{T, \epsilon}\left(r_{j}(\theta, \sigma)\right) \overline{e(\langle\theta, A\rangle)} d \theta .
$$


Precisely as in [PS] the integral in (1.11) will be calculated by the method of stationary phase. We note for emphasis that $r_{j}(\theta, \sigma)$ are the parameters related to the eigenvalues $\lambda_{j}(\theta, \sigma)$ of $\Delta$ on $V(\theta, \sigma)$ by (1.8)(ii).

As in [PS], the main contribution to $I_{T, \epsilon}$ as $T \rightarrow \infty$ comes from the lowest eigenvalue term. The other terms are of exponentially lower order. In fact, if we let $\epsilon$ depend on $T$ as in [PS], and if we set:

$$
I_{T}(C, A)=\sum_{\substack{L ; \\ L(\hat{\gamma})=A \\ \mathrm{Fr}=A}} a_{\gamma}
$$

then, following [PS], we get:

$$
I_{T}(C, A)=\frac{|C|}{|G|} \sum_{\sigma \in \hat{G}} \bar{\chi}_{\sigma}(C) \int_{\Theta} e^{-i r_{0}(\theta, \sigma) T} \frac{\overline{e(\langle A, \theta\rangle)}}{-i r_{0}(\theta, \sigma)} d \theta+O\left(e^{\nu T}\right)
$$

with $\nu<\frac{1}{2}$.

Still following [PS], the asymptotics of the integral in (1.13) are controlled by the critical points of the phase function $r_{0}(\theta, \sigma)$. In fact, $r_{0}(\theta, \sigma)$ is purely imaginary, so that the critical points at $\theta$ with $\lambda_{0}(\theta, \sigma)=0$ will determine $I_{T}(C, A)$ up to an exponentially smaller error. Thus, the key critical points $C P_{0}$ are given by:

$$
C P_{0}=\left\{\theta: \lambda_{0}(\theta, \sigma)=0\right\} .
$$

But clearly $\lambda_{0}\left(\theta_{0}, \sigma\right)=0$ if and only if $\sigma \otimes \chi_{\theta_{0}}$ is the trivial representation. This forces $\sigma$ to have degree 1 and hence to be a character of $G /[G, G]$. Viewing $(G /[G, G])^{\wedge}$ as a set $\left\{\chi_{\theta_{j}}\right\}$ of characters of $\Gamma$, it follows that

$$
C P_{0}=\left\{\theta_{j}: \bar{\chi}_{\theta_{j}} \in(G /[G, G])\right\} \text {. }
$$

Furthermore, the stationary phase expansions of the integrals in (1.13) around the $\theta_{j} \in C P_{0}$ will all have the same coefficients as in [PS]. This follows at once by a linear change of variables. We can therefore follow [PS] through to the end. If we set:

$$
\begin{aligned}
\pi(A, T) & ={ }^{\#}\left\{\hat{\gamma}: L_{\hat{\gamma}} \leq T, H(\hat{\gamma})=A\right\}, \\
\pi(A, C, T) & ={ }^{\#}\left\{\hat{\gamma}: L_{\hat{\gamma}} \leq T, H(\hat{\gamma})=A, \operatorname{Fr}_{\hat{\gamma}}=C\right\} .
\end{aligned}
$$

Then we have:

$$
\frac{\Pi(A, C, T)}{\Pi(A, T)}=\frac{|C|}{|G|} \sum_{\sigma \in(G /[G, G])^{\wedge}} \bar{\chi}_{\sigma}(C) \chi_{\sigma}(A)+O\left(T^{-1}\right) .
$$

This is the precise statement of the theorem.

It is immediate that the probability of a prime from $A$ having Frobenius class $C$ is equal to $(|C| / \mid[[G, G] \mid) \delta(C, A, G /[G, G])$, where $\delta(C, A, G /[G, G])$ equals 1 when $A$ and $C$ project to the same element in $G /[G, G]$, and zero otherwise. When $G$ is Abelian, this probability is 0 or 1 according as $A_{G}$, the projection of $A$ to $G$, equals $C$ or not. When $G$ is perfect, the probability is always $|C| /|G|$. 


\section{REFERENCES}

[AS] Adachi-Sunada, Geodesics in homology classes (preprint).

[PS] R. Phillips and P. Sarnak, Geodesics in homology classes, Duke Math. J. 55 (1987).

[Sa] P. Sarnak, Thesis, Stanford University, 1980.

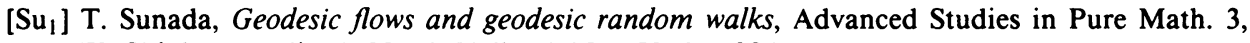
(K. Shiohama, editor), North-Holland, New York, 1984.

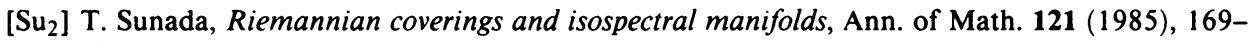
186.

Mathematics Department, Johns Hopkins University, Baltimore, Maryland 21215

Current address: c/o Mathematical Sciences Research Institute, 1000 Centennial Drive, Berkeley, California 94720 